\title{
ENGULFING AND SUBGROUP SEPARABILITY FOR HYPERBOLIC GROUPS
}

\author{
D. D. LONG
}

\begin{abstract}
If a group is subgroup separable, otherwise known as locally extended residually finite or LERF, one can pass from immersions to embeddings in some finite covering space. We show that a certain 'engulfing' property gives subgroup separability for a large and useful class of subgroups of hyperbolic 3-manifold groups.
\end{abstract}

1. Introduction. In geometric topology, one of the most useful properties that a group can have is that it is subgroup separable, otherwise known as locally extended residually finite or LERF. One of the reasons that the LERF property is so powerful is that it enables one to pass from immersions to embeddings in some finite covering space. Unfortunately, this strong property is known for only a relatively small class of groups. (The reader is referred to the results of [6] for a summary of what is known in general.) In particular, as remarked in [8], there seem to be very few hyperbolic 3-manifold groups for which it is known. In this paper, we shall show that a certain 'engulfing' property gives subgroup separability for a large and useful class of subgroups of hyperbolic 3-manifold groups.

We begin with some definitions and notation. Let $G$ be a group and $H$ a finitely generated subgroup. Then $G$ is said to be $H$-subgroup separable if given any element $g \in G$, which does not lie in $H$, there is a proper subgroup of finite index, $K<G$, with $H \leq K$ and $g$ not lying in $K$. A group which is $\{e\}$-subgroup separable, where $\{e\}$ is the trivial subgroup, is more usually called residually finite.

A group which is $H$-subgroup separable for every finitely generated subgroup is said to be subgroup separable or LERF.

In order to state our results concisely, we introduce some nonstandard notation at this point. Given a subgroup $H \leq G$, we define $H^{*}$ by $H^{*}=\bigcap\{K \mid H \leq K \leq G$ and $K$ has finite index in $G$ \}. In these words, a group is $H$-subgroup separable if and only if $H^{*}=H$. For later use, we also introduce the notion of virtually $H$-separable, which will mean that the index of $H$ in $H^{*}$ is finite. We shall show that for many geometric purposes, virtual separability is as good as separability.

One of the difficulties of dealing with the LERF property is that there often appears to be nowhere to start, that is, it is conceivable that a finitely generated proper subgroup could be contained in no proper subgroups of finite index at all. (Equivalently, $H^{*}=G$, even though $H<G$. In fact, it is easy to see that the ideas of [5] easily construct infinitely generated such $H$ inside any hyperbolic group. This is sketched in §3.) We have been unable to circumvent this problem in general, so we shall hypothesise it away:

Received by the editors April 20, 1987 and, in revised form, June 17, 1987.

1980 Mathematics Subject Classification (1985 Revision). Primary 57M05; Secondary 57M10. 
DEFINITION. A group $G$ is said to have the engulfing property if given a finitely generated subgroup $H \leq G$, there is some proper subgroup $K$, of finite index in $G$, and with $H \leq K<G$.

In the notation which has been established above, $G$ has the engulfing property if for every finitely generated $H<G$, we have that $H^{*}<G$. This is clearly a very much weaker property than LERF, and will be the starting point for our results.

The context in which we shall work is that of hyperbolic 3-manifold groups, and an examination of the extent to which the engulfing property implies LERF. Accordingly, we shall reserve the symbol $\Gamma$ for discrete, torsion-free subgroup of $P S L_{2}(\mathbf{C})$, with the property that $\mathbf{H}^{3} / \Gamma$ is a closed 3-manifold. If $H$ is a subgroup of $\Gamma$, then associated to $H$ is a partition of the 2-sphere at infinity, $S_{\infty}^{2}$, into two subsets; the limit set, $\Lambda H$ and the domain of discontinuity, $\Omega H$. We shall also refer to the convex hull $C(\Lambda H)$ of $\Lambda H$, which is the intersection of all the hyperbolic half spaces which contain $\Lambda H$. For technical reasons, it is also useful to consider the $\varepsilon$-neighbourhood of the convex hull, $N_{\varepsilon}(C(\Lambda H)) . H$ acts on its convex hull as a group of covering translations and is said to be geometrically finite if the quotient $N_{\varepsilon}(C(\Lambda H)) / H$ has finite volume. A good general reference for this material can be found in [9].

We can now state our.main result:

THEOREM 2.7. Let $\Gamma$ be the fundamental group of a closed, hyperbolic 3manifold. Suppose that $\Gamma$ has the engulfing property for those finitely generated subgroups $H$ with $\Lambda H<S_{\infty}^{2}$.

Then every geometrically finite subgroup $H \leq \Gamma$ is virtually $H$-separable.

From this we can deduce the following geometric result. If such a $\Gamma$ contains a surface group which is geometrically finite then the usual arguments for LERF groups show that we can replace an immersion with an embedding in a finite covering. With a small amount of further argument it follows that:

COROLlARY 2.9. Let $\Gamma$ be as in the statement of 2.7. Then if $\Gamma$ contains a surface group, the manifold $\mathbf{H}^{3} / \Gamma$ is virtually Haken.

The complete argument for this will be presented in 2.9. In particular, we deduce that in the presence of the engulfing property, the existence of an immersed quasifuchaian or totally geodesic surface in $\mathbf{H}^{3} / \Gamma$ implies the existence of an embedded one in some finite covering.

The result of 2.7 follows rather easily from our next result, which is of some independent interest:

THEOREM 2.6. Let $\Gamma$ be as in the statement of 2.7. Then for any finitely generated subgroup $H \leq \Gamma$, we have $\Lambda H^{*}=\Lambda H$.

As is well known, the LERF property is closely related to the question of whether a Haken manifold is finitely covered by a manifold with positive first Betti number. We show

THEOREM 2.10. Let $\Gamma$ be as in the statement of 2.7. If the manifold $\mathbf{H}^{3} / \Gamma$ is Haken, then there is a finite covering $M \rightarrow \mathbf{H}^{3} / \Gamma$ for which $\operatorname{rank}\left(H_{1}(M ; \mathbf{Q})\right)>0$. 
We also briefly examine the engulfing property. For example it is shown that engulfing implies residual finiteness. We also have the result that engulfing is well behaved for subgroups and for supergroups of finite index. More precisely:

THEOREM 3.1. Let $\Gamma_{1}$ and $\Gamma_{2}$ be the fundamental group of closed, hyperbolic manifolds, with $\Gamma_{1}$ containing $\Gamma_{2}$ as a subgroup of finite index.

Then $\Gamma_{1}$ has the engulfing property for all finitely generated subgroups $H$ with $\Lambda H<S^{2}$ if and only if $\Gamma_{2}$ does.

Most of the results of this paper apply to manifolds with boundary, if the obvious modifications are made to the statements, however we shall work exclusively in the closed case. Similarly, many of the hyperbolic geometry results are independent of dimension, though this too will be suppressed.

2. Main results. In this section we shall prove our main results.

Some of the proofs below become longer or false if the subgroup $H$ is elementary; that is, it contains an abelian subgroup of finite index. Accordingly, we shall adopt the convention that throughout this paper, the word 'subgroup' is to mean 'nonelementary subgroup'.

We begin by introducing some further notation. Recall that if $\gamma \in \Gamma$, its action on $\mathbf{H}^{3}$ is hyperbolic, that is, it acts as translation along some geodesic, the endpoints of which are fixed points of the induced action on $S_{\infty}^{2}$. These fixed points will be denoted by $\partial \gamma$. Suppose that $U_{+}$is a neighbourhood in $S_{\infty}^{2}$ of the source, and $U_{-}$ is some neighbourhood of the sink, which is disjoint from $U_{+}$. Then we shall say that the pair $\left(U_{+}, U_{-}\right)$is absorbing for $\gamma$ if:

(a) Given any $p \in S_{\infty}^{2} \backslash U_{-}$, then $\gamma(p) \in U_{+}$.

(b) Given any $p \in S_{\infty}^{2} \backslash U_{+}$, then $\gamma^{-1}(p) \in U_{-}$.

Observe that any pair of disjoint neighbourhoods of the fixed points is absorbing for $\gamma^{k}$, for some sufficiently large $k$.

The only slightly nonstandard fact about limit sets we shall need is

LEMMA 2.1. Let $H \leq P S L_{2}(\mathbf{C})$. Then $\Lambda H$ is the closure in $S_{\infty}^{2}$ of the set $\{\partial \gamma \mid \gamma \in H\}$.

PROOF. Since by convention the subgroup $H$ is nonelementary, its limit set is well known to be the minimal closed $H$-invariant subset of $S_{\infty}^{2}$. It is clear that the set $\{\partial \gamma \mid \gamma \in H\}$ lies inside $\Lambda H$, and on the other hand it is clearly invariant. Hence its closure must be the limit set.

We now accumulate some general facts about the behaviour of the *-operator. None of these are special to the hyperbolic situation.

LEMMA 2.2. For any subgroup $H \leq G,\left(H^{*}\right)^{*}=H^{*}$.

ProOF. If $K$ is a subgroup of finite index in $G$, then $K \geq H$ if and only if $K \geq H^{*}$. This proves the result.

REMARK. Thus for any subgroup $H$, the group $H^{*}$ is genuinely separable (that is, if we set $A=H^{*}$, then $A=A^{*}$ ) provided that it is a proper subgroup.

LEMMA 2.3. (a) Suppose that $N \triangleleft H$ is a subgroup of finite index. Then there is an integer $t$ (in fact, $[H: N]$ will do), so that if $\varphi \in H^{*}$, then $\varphi^{t} \in N^{*}$.

(b) Suppose that $N \triangleleft H$. Then $N^{*} \triangleleft H^{*}$. 
ProOF. (a) Let $K$ be a subgroup of finite index in $G$, with $K \geq N$. We need to show that if $\varphi \in H^{*}$ then $\varphi^{t} \in K$, where $t=[H: N]$.

There is an action of $H$ on $G$ by conjugation and since $K$ is of finite index, it has only finitely many $H$-conjugates. Intersecting all these gives a $K^{\prime}$ also of finite index in $G$, which is normalised by $H$. Moreover, since $N$ was normal in $H$, we have $K \geq K^{\prime} \geq N$.

Since $K^{\prime}$ is normalised by $H$, the subgroup generated by $K^{\prime}$ and $H$ is the group $H \cdot K^{\prime}$. Then by the standard isomorphism theorems, we have $\left[H \cdot K^{\prime}: K^{\prime}\right]=$ $\left[H: H \cap K^{\prime}\right]=[H: N] /\left[H \cap K^{\prime}: N\right]$. Thus the order of the group $H \cdot K^{\prime} / K^{\prime}$ divides $[H: N]$, and so any element of this quotient group has order dividing $[H: N]$.

If now $\varphi$ is any element of $H^{*}$, then $\varphi \in H \cdot K^{\prime}$, since this is a subgroup of finite index which contains $H$. Hence $\varphi^{t} \in K^{\prime} \leq K$, as required.

(b) We shall never actually use this fact, and since it has a similar proof, it is left as an exercise.

COROLlaRY 2.4. If $N \triangleleft H$ is normal and of finite index, then $\Lambda N^{*}=\Lambda H^{*}$.

PROOF. It is clear that $H^{*} \geq N^{*}$, so that $\Lambda H^{*} \supset \Lambda N^{*}$. Conversely, part (a) in the above result, together with 2.1 implies the other inclusion.

REMARK. In fact, the result of 2.3(b) shows that the hypothesis of finite index is not necessary, but we shall never appeal to this fact.

Before embarking on the proof of our main result, we prove a preliminary theorem, which illustrates some of the key ideas involved. We shall actually prove more results concerning the engulfing property in $\S 3$, however it seems appropriate to observe at this point that in our situation engulfing actually implies residual finiteness. (Of course, this result is not new-subgroups of $P S L_{2}(\mathbf{C})$ are well known to be residually finite by a result of $\mathrm{Mal}^{\prime} \mathrm{cev}$. Our motivation is that a geometric proof of engulfing would yield a geometric proof of the residual finiteness of hyperbolic groups.)

COROLLARY 2.5. Let $\Gamma$ be the fundamental group of a closed, hyperbolic manifold. Suppose that $\Gamma$ has the engulfing property. Then $\Gamma$ is residually finite.

Proof. Consider the subgroup $\{e\}^{*}$, where $\{e\}$ is the trivial subgroup of $\Gamma$. Then $\Gamma$ is residually finite if and only if $\{e\}^{*}=\{e\}$. If this were not the case, then $\{e\}^{*}$ would be a nontrivial normal subgroup of $\Gamma$, and so by a standard argument (see for example [9, Corollary 8.1.3]) $\Lambda\{e\}^{*}=S^{2}$. We shall use this fact to derive a contradiction.

Suppose that $\Gamma$ is generated by $\gamma_{1}, \ldots, \gamma_{n}$. Choose elements $x_{1}, \ldots, x_{n}$ which lie in $\{e\}^{*}$ and so that:

(i) $\partial x_{a} \neq \partial x_{b}$ if $1 \leq a \neq b \leq n$,

(ii) $\partial x_{a} \neq \partial \gamma_{a}, 1 \leq a \leq n$.

The second condition ensures that the four points $\partial x_{a} \cup \gamma_{a}\left(\partial x_{a}\right)$ are all distinct for any $a$, since in a closed, hyperbolic 3-manifold group, fixed points of hyperbolics coincide or are disjoint. Hence we may find disjoint neighbourhoods of $\partial x_{a}$, call these $\left(U_{a}\right)^{+}$and $\left(U_{a}^{-}\right)$, with:

(i) $\left(U_{a}\right)^{+} \cup\left(U_{a}\right)^{-}$is disjoint from $\left(U_{b}\right)^{+} \cup\left(U_{b}\right)^{-}$if $a \neq b$.

(ii) $\left(U_{a}\right)^{+} \cup\left(U_{a}\right)^{-}$is disjoint from $\gamma_{a}\left(\left(U_{a}\right)^{+} \cup\left(U_{a}\right)^{-}\right)$for each $a$. 
By replacing each $x_{a}$ by a power $K>0$ if necessary (and renaming) we can arrange that the pair $\left(\left(U_{a}\right)^{+},\left(U_{a}\right)^{-}\right)$is absorbing for each $x_{a}$. Set $\varphi_{a}=x_{a} \gamma_{a} x_{a}$.

Then we claim that the pair $\left(\left(U_{a}\right)^{+},\left(U_{a}\right)^{-}\right)$is in fact absorbing for $\varphi_{a}$. For if $P$ is a point in $S^{2}$ not lying in $\left(U_{a}\right)^{-}$, then $x_{a}(P)$ lies in $\left(U_{a}\right)^{+}$since the pair was absorbing for $x_{a}$. Then $\gamma_{a} x_{a}(P)$ lies in $\gamma_{a}\left(U_{a}\right)^{+}$which is disjoint from $\left(U_{a}\right)^{-}$by (ii). Hence $x_{a} \gamma_{a} x_{a}(P)$ lies in $\left(U_{a}\right)^{+}$, as required. The result for $\varphi_{a}^{-1}$ follows in a similar fashion.

We claim that the group generated by $\varphi_{1}, \ldots, \varphi_{n}$ is free, and in fact has limit set contained in the closure of $\left(U_{1}\right)^{+} \cup\left(U_{1}\right)^{-} \cup \cdots \cup\left(U_{n}\right)^{+} \cup\left(U_{n}\right)^{-}$. This follows the usual Schottky group type argument following the track of one point. This is done in 2.6, so we suppress it here. In particular, since $\Lambda N<S^{2}, N=\operatorname{grp}\left\{\varphi_{1}, \ldots, \varphi_{n}\right\}$ is a proper subgroup of infinite index in $\Gamma$.

Since by hypothesis $\Gamma$ engulfs such subgroups, there is a subgroup $K<\Gamma$ of finite index, with $N \leq K$. But $K$ must contain $\{e\}^{*}$, and hence $x_{1}, \ldots, x_{n}$. Since $K$ contains the $\varphi_{i}$ 's, it must contain $\gamma_{1}, \ldots, \gamma_{n}$. But this set generates $\Gamma$, a contradiction. Hence $\{e\}^{*}=\{e\}$, as required.

We now give the proof of our main result. It is similar in outline to the above result, but differs in some of the details, since the structure of the subgroup in question can be a little more complicated.

THEOREM 2.6. Let $\Gamma \leq P S L_{2}(\mathbf{C})$ be the fundamental group of a closed, hyperbolic 3-manifold. Suppose that $\Gamma$ has the engulfing property for those finitely generated subgroups $P$ with $\Lambda P<S^{2}$.

Then for all finitely generated $H \leq \Gamma$, we have $\Lambda H^{*}=\Lambda H$.

ProOF. Let $H \leq \Gamma$ be a subgroup. Then if $\Lambda H=S^{2}$, the result is clear, since $H^{*} \geq H$, and so $\Lambda H^{*}=S^{2}$. Hence we can suppose that $\Lambda H<S^{2}$.

Choose some fixed finite generating set $\gamma_{1}, \ldots, \gamma_{n}$ for $\Gamma$. In search of a contradiction, we assume that $\Lambda H^{*}>\Lambda H$. Then by Lemma 2.1, there is a hyperbolic element $\alpha \in H^{*}$ with at least one endpoint in $\Lambda H^{*} \backslash \Lambda H$, say that this is the attracting endpoint. Notice that if $\gamma$ is any hyperbolic, the fixed points of $\alpha \cdot \gamma \cdot \alpha^{-1}$ are $\alpha(\partial \gamma)$. So if $\gamma \in H^{*}$, for sufficiently large $K$, the fixed points of $\alpha^{K} \cdot \gamma \cdot \alpha^{-K}$ lie inside $\Lambda H^{*} \backslash \Lambda H$.

It follows that we can find hyperbolics $\beta_{1}, \ldots, \beta_{n} \in H^{*}$ with $\partial \beta_{i}$ lying in $\Lambda H^{*} \backslash \Lambda H$ for all $i$. Further, we arrange that $\partial \beta_{i}$ is not $\partial \gamma_{i}$ for any $i$.

Choose some compact set $C$ lying in $S^{2} \backslash \Lambda H$ with $\partial \beta_{i} \in \operatorname{int} C$ for each $i$. The group $H$ acts properly discontinuously on $S^{2} \backslash \Lambda H$, so there are only a finite number of elements of $H, h_{1}, \ldots, h_{m}$, which have $h_{t}(C) \cap C \neq \varnothing$. The group $H$ is linear, so it is residually finite and there is therefore a normal subgroup of finite index $N \triangleleft H$ which contains none of the elements $h_{1}, \ldots, h_{m}$. By Lemma 2.3, we may replace each $\beta_{i}$ by some power and suppose that $\beta_{i} \in N^{*}$, for each $i$.

Choose a pair of disjoint neighbourhoods $\left(U_{i}, V_{i}\right)$ of $\partial \beta_{i}$ which have the properties that:

(a) $\operatorname{cl}\left(U_{i}\right), \operatorname{cl}\left(V_{i}\right) \subset \operatorname{int}(C)$.

(b) The sets $\gamma_{i}\left(U_{i}\right) \cup \gamma_{i}\left(V_{i}\right)$ and $U_{i} \cup V_{i}$ are disjoint for $1 \leq i \leq n$.

(c) $\operatorname{cl}\left(U_{i}\right) \cup \operatorname{cl}\left(V_{i}\right)$ is disjoint from $\operatorname{cl}\left(U_{t}\right) \cup \operatorname{cl}\left(V_{t}\right)$ for $1 \leq i \neq t \leq n$. 
By replacing $\beta_{i}$ by some further positive power if necessary, and renaming, we arrange that the pair $\left(U_{i}, V_{i}\right)$ is absorbing for $\beta_{i}$.

Set $\varphi_{i}=\beta_{i} \gamma_{i} \beta_{i}$. Exactly as in the proof of 2.5 , the pair $\left(U_{i}, V_{i}\right)$ is absorbing for $\varphi_{i}$

From this it follows that $\operatorname{grp}\left\{\varphi_{1}, \ldots, \varphi_{n}\right\}$ is free of rank $n$. For if $\Theta$ is any reduced word in the $\varphi_{i}$ 's, one can verify explicitly that any point which lies in the set $S^{2} \backslash$ closure $\left(U_{1} \cup V_{1} \cup \cdots \cup U_{n} \cup V_{n}\right)$ is moved into the set $U_{1} \cup V_{1} \cup \cdots \cup U_{n} \cup V_{n}$. Hence the word $\Theta$ cannot be the identity.

We now consider the subgroup of $\Gamma$ generated by $\varphi_{1}, \ldots, \varphi_{n}$ and $N$. Call this subgroup $A$. Our next claim is that

$$
\Lambda A \subset \operatorname{cl}\left(S^{2} \backslash C\right) \cup \operatorname{cl}\left(U_{1}\right) \cup \operatorname{cl}\left(V_{1}\right) \cup \cdots \cup \operatorname{cl}\left(U_{n}\right) \cup \operatorname{cl}\left(V_{n}\right),
$$

and in particular that $\Lambda A<S^{2}$.

Since the limit set is determined by examination of the orbit of any point, this claim may be verified by consideration of the action of any element of $A$ on a point $P$ in $C \backslash \operatorname{cl}\left(U_{1}\right) \cup \operatorname{cl}\left(V_{1}\right) \cup \cdots \cup \operatorname{cl}\left(U_{n}\right) \cup \operatorname{cl}\left(V_{n}\right)$. If we choose any word in $A$, it has the form $n_{1} f_{1} n_{2} \cdots n_{t} f_{t}$, where each $n_{i} \in N$ and $f_{i} \in \operatorname{grp}\left\{\varphi_{1}, \ldots, \varphi_{n}\right\}$ and apart from possibly $n_{1}$ or $f_{t}$, none of these elements is the identity. Let us deal with the case that $f_{t}$ is not the identity - the other cases are similar. Then since $P$ does not lie in $\operatorname{cl}\left(U_{1}\right) \cup \operatorname{cl}\left(V_{1}\right) \cup \cdots \cup \operatorname{cl}\left(U_{n}\right) \cup \operatorname{cl}\left(V_{n}\right)$, the point $f_{t}(P)$ lies in $U_{1} \cup V_{1} \cup \cdots \cup U_{n} \cup V_{n}$. This set is contained in $C$, so that $n_{t} f_{t}(P)$ lies in $S^{2} \backslash C$. Since this set is disjoint from $U_{1} \cup V_{1} \cup \cdots \cup U_{n} \cup V_{n}$, we see that $f_{t-1} n_{t} f_{t}(P)$ lies in $U_{1} \cup V_{1} \cup \cdots \cup U_{n} \cup V_{n}$. And so on. The point $P$ is always either moved out of $C$, or into the set $U_{1} \cup V_{1} \cup \cdots \cup U_{n} \cup V_{n}$. Thus its only possible accumulation points lie in the set $\operatorname{cl}\left(S^{2} \backslash C\right) \cup \operatorname{cl}\left(U_{1}\right) \cup \operatorname{cl}\left(V_{1}\right) \cup \cdots \cup \operatorname{cl}\left(U_{n}\right) \cup \operatorname{cl}\left(V_{n}\right)$, as was claimed.

The subgroup $N$ is finitely generated, since it is a subgroup of finite index in $H$, hence $A$ is finitely generated. By hypothesis, the group $\Gamma$ engulfs such subgroups, so there is a proper subgroup $K<G$, which has finite index, and has $A \leq K$. Therefore $K \geq N^{*}$, and hence $K$ contains the elements $\beta_{1}, \ldots, \beta_{n}$. But this means that $K$ contains the elements $\gamma_{1}, \ldots, \gamma_{n}$ which generate $\Gamma$. This contradiction completes the proof.

THEOREM 2.7. Suppose that $\Gamma$ is the fundamental group of a closed, hyperbolic 3-manifold and that it has the engulfing property for finitely generated subgroups $H$ with $\Lambda H<S^{2}$.

If $H$ is a geometrically finite subgroup of $\Gamma$, then it is virtually $H$-separable.

ProOF. By the result of 2.6, $\Lambda H^{*}=\Lambda H$. This means that the group $H^{*}$ preserves the convex hull $C(\Lambda H)$. This action is properly discontinuous, so the map $C(\Lambda H) \rightarrow C(\Lambda H) / H^{*}$ is a covering map. By definition, the manifold $C(\Lambda H) / H$ has finite volume. (Strictly, we should work with an $\varepsilon$-neighbourhood, but this does not change the argument.) Then we have a covering $C(\Lambda H) / H \rightarrow C(\Lambda H) / H^{*}$ which exhibits the base as a 3-manifold of finite volume. Hence the index $\left[H^{*}: H\right]$ is finite, it is in fact the volume ratio $\operatorname{vol}(C(\Lambda H) / H) / \operatorname{vol}\left(C(\Lambda H) / H^{*}\right)$. This shows virtual $H$-separabilty as required.

Let us note that since by $2.2,\left(H^{*}\right)^{*}=H^{*}$, the subgroup $H^{*}$ is genuinely subgroup separable. In the geometrically finite case, this entails only finite extension. 
It is this fact which is the basis of the following applications, which show that despite the fact that 2.7 does not give the full strength of LERF, for many geometric purposes this weaker property is sufficient.

COROLlaRY 2.8. Suppose that $\Gamma$ is as in the statement of 2.6.

If $H \leq \Gamma$ is a geometrically finite surface group corresponding to a closed orientable surface $F$ which is embedded in $\mathbf{H}^{3} / \Gamma$, then $H$ is genuinely separable.

ProOF. By the result of 2.7 , the index $\left[H^{*}: H\right]$ is finite, and since $H$ comes from a closed orientable surface, the results in the $I$-bundles chapter in [4], imply that this index must be 1 or 2 . If the index is 1 , we are done, so we must rule out the other case.

If the index is 2 , the group $H^{*}$ corresponds to $F^{\prime}$, a one-sided (and thus nonorientable) surface embedded in $\mathbf{H}^{3} / \Gamma$. Moreover, $F$ is the boundary of a regular neighbourhood of $F^{\prime}$. However there is a degree two covering $\mathbf{H}^{3} / \Gamma$ made by taking two copies of $M \backslash N\left(F^{\prime}\right)$ and pasting these together along the copies of $F$. This covering corresponds to a subgroup of index two in $\Gamma$ which contains $H$ but not $H^{*}$. This is a contradiction and we deduce that $\left[H^{*}: H\right]=1$.

COROLlary 2.9. Let $\Gamma$ be as in the statement of 2.6. Suppose that the manifold $M=\mathbf{H}^{3} / \Gamma$ contains a subgroup corresponding to a closed, orientable surface. Then $M$ is virtually Haken.

PROOF. There are two cases, depending whether or not the surface group $H \leq \Gamma$ is geometrically finite.

(a) $H$ is geometrically infinite.

Several topologists have observed that it follows from the work of Bonahon [2] and Thurston that a geometrically infinite surface group is virtually a fibre. We shall therefore only outline the proof. This version was shown to the author by Peter Scott.

If the group $H$ is geometrically infinite, then [2] implies that there is a sequence of embedded pleated surfaces going off to either of the ends of $\mathbf{H}^{3} / H$. If we project into the closed manifold $\mathbf{H}^{3} / \Gamma$ the compactness of the space of pleated surfaces [9] shows that we can find a pair of surfaces which are very far apart in $\mathbf{H}^{3} / H$, yet very close (and hence homotopic) in $\mathbf{H}^{3} / \Gamma$. Thus there is an arc in the covering connecting one of these surfaces to the other in $\mathbf{H}^{3} / H$. This arc can be chosen so that after projection it corresponds to a closed loop representing an element $t$ of $\Gamma$ which normalises the surface group $H$. The subgroup generated by $t$ and $H$ gives a finite covering of $\mathbf{H}^{3} / \Gamma$ which is a bundle.

(b) $H$ is geometrically finite.

By the result of $2.7,\left[H^{*}: H\right]$ is finite, so the group $H^{*}$ is also a surface group. The easiest way to see this is to observe that in the covering $C(\Lambda H) / H \rightarrow C(\Lambda H) / H^{*}$, the total space has the fundamental group of a closed surface group, namely $H$. Now by Theorem 10.6 of $[4], H^{*}$ must also be a (possibly nonorientable) surface group.

Moreover, $H^{*}$ corresponds to a surface immersed in $M$, for which we have genuine separability. We now apply a standard argument [6] (see below) for obtaining an embedded surface in some finite covering $M_{f}$ of $M$. Exactly as in the proof of 2.8 , we deduce that the surface corresponding to $H^{*}$ is orientable. This two-sided incompressible embedded surface exhibits $M_{f}$ as a Haken manifold. 
For the convenience of the reader unfamiliar with the arguments as those used in [6] (the relevant part of [6] being Lemma 1.6 and the remark following it) we give a sketch. Lift the immersion corresponding to $H^{*}$ to $\mathbf{H}^{3} / H^{*}$. Since $H^{*}$ is geometrically finite, the inclusion of the convex hull quotient $C\left(\Lambda H^{*}\right) / H^{*}$ into $\mathbf{H}^{3} / H^{*}$ induces an isomorphism at the fundamental group level. Recall that the manifold was supposed closed, so that $C\left(\Lambda H^{*}\right) / H^{*}$ is compact. Let $p$ denote the projection $\mathbf{H}^{3} \rightarrow \mathbf{H}^{3} / H^{*}$. Then there is clearly a compact $D$ in $\mathbf{H}^{3}$ with $p(D)=$ $C\left(\Lambda H^{*}\right) / H^{*}$. By proper discontinuity, the set $\{g \in \Gamma \mid g(D) \cap D \neq \varnothing\}$ is finite. Since $H^{*}$ is separable inside $\Gamma$, there is therefore a subgroup $K$ of finite index in $\Gamma$ with the property that $K$ contains $H^{*}$, and that if $g \in \Gamma$ has $g(D)$ meeting $D$, then $g$ lies in $H^{*}$. Then $\mathbf{H}^{3} / K$ embeds both of the surfaces $\partial_{ \pm} C\left(\Lambda H^{*}\right) / H^{*}$ and is therefore a covering with the required properties.

REMARK. Observe that the method of proof promotes a finite extension of the given surface group to an embedding in some finite covering. In particular, quasifuchsian or totally geodesic surfaces can be lifted to an embedding of the same type in a finite covering. In fact, the totally geodesic case can be handled by a separate argument which bypasses engulfing, see the remarks following Theorem 3.2 or [10].

As is remarked in [8], there is a connection between the LERF property and the question of whether a Haken manifold is finitely covered by a manifold with positive first betti number. In the light of our results we can reduce this in the hyperbolic case to a question about the engulfing property. This argument in the case of LERF groups is fairly well known, but we give a sketch here.

THEOREM 2.10. Let $\Gamma$ be the fundamantal group of a closed hyperbolic 3manifold and suppose that $\Gamma$ has the engulfing property for finitely generated subgroups $H$ with $\Lambda H<S^{2}$.

If the manifold $\mathbf{H}^{3} / \Gamma$ is Haken, then there is a finite covering $M \rightarrow \mathbf{H}^{3} / \Gamma$ for which $\operatorname{rank}\left\{H_{1}(M ; \mathbf{Q})\right\}>0$.

ProOF. Since the manifold $A=\mathbf{H}^{3} / \Gamma$ is Haken it contains an embedding of a closed, orientable surface $F$, with the map $\pi_{1}(F) \rightarrow \pi_{1}(A)=\Gamma$ an injection. Let the image of this map be $H$. The results of Thurston, as expounded in [7] imply that we may assume that the group $H$ is quasi-fuchsian (for if it were not it would virtually be a fibre and we would be done), in particular it is geometrically finite. Thus the result of 2.8 implies that $H$ is genuinely separable.

The surface $F$ must separate, or we are finished, so let the components be $C_{ \pm}$. We have two inclusion maps $\pi_{1}(F) \rightarrow \pi_{1}\left(C_{ \pm}\right)$; untwisting any $I$-bundle pieces, we can assume that the subgroup $H$ is of infinite index in each of $\pi_{1}\left(C_{ \pm}\right)$. Choose elements $g_{ \pm} \in \pi_{1}\left(C_{ \pm}\right) \backslash H$; because $H$ is separable in $\Gamma$, there is a $K$ of finite index in $\Gamma$ which contains $H$ and neither of $g_{ \pm}$. Intersecting $K$ with all its $\Gamma$ conjugates, we obtain a normal subgroup of finite index in $\Gamma$, which we denote $N$. Notice that $N$ may no longer contain $H$. Then we claim that the covering $p: \mathbf{H}^{3} / N \rightarrow \mathbf{H}^{3} / \Gamma$ has the property that some component of the preimage of $F$ does not separate.

The argument is this: Fix one component of $p^{-1}\left(C_{+}\right)$, call this $p^{-1}\left(C_{+}\right)_{0}$ and consider the covering $p^{-1}\left(C_{+}\right)_{0} \rightarrow C_{+}$. This is defined by restricting the homomorphism $\Gamma \rightarrow \Gamma / N$ to a homomorphism $\pi_{1}\left(C_{+}\right) \rightarrow \pi_{1}\left(C_{+}\right) \cdot N / N$. The subgroup $H$ cannot surject onto this quotient since its image lies inside $K / N$ which does not 
contain the $g_{+}$coset. Hence $F$ has more than one preimage in $p^{-1}\left(C_{+}\right)_{0}$. By regularity, this then applies to each component of the $p$-preimage of $C_{+}$, and similarly to $C_{-}$. Since the covering of $\mathbf{H}^{3} / \Gamma$ by $\mathbf{H}^{3} / N$ is required to be connected, it now follows easily that at least one, and hence, every component of the preimage of $F$ is nonseparating in $\mathbf{H}^{3} / N$.

We conclude this section with a slight digression. To place this in context, recall that if $G$ is a group, then the Frattini subgroup of $G$, usually written $\Phi(G)$, is the intersection of all the maximal subgroups of $G$. There has been some interest (see [1]) in the Frattini subgroups of knot complements and 3-manifolds.

Our next result covers some of the cases not covered in [1]. Notice that it is not necessary to assume engulfing here.

THEOREM 2.11. Let $\Gamma$ be the fundamental group of a closed, hyperbolic 3manifold. Then $\Phi(\Gamma)=\{e\}$.

SKETCH PROOF. As in the proof of 2.5 , if this were not the case, $\Lambda \Phi(\Gamma)=S^{2}$ and we construct the same subgroup $N=\operatorname{grp}\left\{\varphi_{1}, \ldots, \varphi_{n}\right\}$. Now by Zorn's lemma, there is a maximal subgroup $M<\Gamma$, with $N \leq M$. Then $M \geq \Phi(\Gamma)$, so we conclude as in 2.5 that $M$ contains a generating set, which is the same contradiction.

3. The engulfing property. In this section, we briefly explore the engulfing property. As has already been observed in Corollary 2.5, in the context of hyperbolic 3-manifold groups, this property is stronger than residual finiteness. It is nevertheless a good deal weaker than the LERF property and of course, the latter property easily implies it.

Let us make some trivial observations. The first is that it is always possible to engulf cyclic groups. A construction which shows this is the following: If $g \in \Gamma$ is not trivial, choose any nontrivial element of the commutator subgroup of $\Gamma$, call this $c$, say. By residual finiteness, find a normal subgroup of finite index $K \triangleleft \Gamma$ which does not contain $c$. Then the quotient group $\Gamma / K$ is not abelian, since $c$ does not die. In particular, this quotient group is not cyclic, so the subgroup $\operatorname{grp}\{g, K\}$ is a proper subgroup of finite index which contains $g$. In fact, the author has shown that cyclic subgroups in closed, hyperbolic 3-manifold groups are virtually separable. This is discussed in $[\mathbf{1 0}]$.

In the proof of 2.6 , it was actually only necessary to engulf subgroups $H$ with $\Lambda H<S^{2}$. Let us observe that in the hyperbolic context this property is well behaved for subgroups and for supergroups of finite index. (The analogous proposition for general groups is false.)

THEOREM 3.1. Let $\Gamma_{1}$ and $\Gamma_{2}$ be the fundamental group of a closed, hyperbolic manifolds, with $\Gamma_{1}$ containing $\Gamma_{2}$ as a subgroup of finite index.

Then $\Gamma_{1}$ has the engulfing property for all finitely generated subgroups $H$ with $\Lambda H<S^{2}$ if and only if $\Gamma_{2}$ does.

Proof. (a) Suppose $\Gamma_{1}$ has the engulfing property for these subgroups. We wish to show the property for $\Gamma_{2}$.

Accordingly, let $H<\Gamma_{2}$ be such a subgroup. Since $\Lambda \Gamma_{2}=S^{2}$, we may find a $\varphi \in \Gamma_{2}$ with $\partial \varphi$ not lying in $\Lambda H$. By the result of 2.6 applied to $\Gamma_{1}, \varphi$ does not lie in $H^{*}$ so that there is a subgroup $K$, of finite index in $\Gamma_{1}$ with $H<K<\Gamma_{1}$, and $\varphi$ not in $K$. Then $K \cap \Gamma_{2}$ engulfs $H$ inside $\Gamma_{2}$. 
(b) Now suppose that $\Gamma_{2}$ has the engulfing property and choose such an $H \leq \Gamma_{1}$. By using the conjugation action of $H$ on $\Gamma_{1}$ there is a $\Gamma_{3} \leq \Gamma_{2}$ of finite index which is normalised by $H$. The result of the first paragraph shows that $\Gamma_{3}$ has the engulfing property.

Consider now the subgroup $H \cap \Gamma_{3}$. This is a normal subgroup of finite index in $H$, so it is finitely generated and by 2.6 we have $\Lambda\left(H \cap \Gamma_{3}\right)^{*_{3}}=\Lambda\left(H \cap \Gamma_{3}\right)$. (Here we use the $*_{i}$-operator to refer to the subgroups of finite index in $\Gamma_{i}$.) However since $\Gamma_{3}$ has finite index in $\Gamma_{1}$, it is clear that $\Lambda\left(H \cap \Gamma_{3}\right) \subset \Lambda\left(H \cap \Gamma_{3}\right)^{*_{1}} \subset \Lambda\left(H \cap \Gamma_{3}\right)^{*_{3}}$. Thus these are all equalities. But the result of 2.4 (which was purely formal and did not require engulfing) shows that $\Lambda\left(H \cap \Gamma_{3}\right)^{*_{1}}=\Lambda H^{*_{1}}$. In particular, we deduce that $\Lambda H^{*_{1}}$ is not the whole of $S^{2}$, implying that $H$ can be engulfed in $\Gamma_{1}$.

Let us now note that if we drop finite generation in the engulfing condition, there are proper subgroups in any hyperbolic group which cannot be engulfed. This construction is inspired by the result of Margulis-Soifer contained in [5].

THEOREM 3.2. Let $\Gamma$ be the fundamental group of a closed hyperbolic 3-manifold. Then $\Gamma$ contains a subgroup $H$, with $\Lambda H<S^{2}$ and $H^{*}=\Gamma$.

SKETCH PROOF. The group $\Gamma$ contains only a countable number of normal subgroups of finite index; let $K_{1}, K_{2}, \ldots$ be some enumeration. It suffices to show that we can construct an $H$ with the property that for every $i, H$ maps onto $\Gamma / K_{i}$. For if such an $H$ could be engulfed in some subgroup of finite index, $K<\Gamma$, then we can pass to a normal subgroup of finite index $K^{\prime} \leq K$. But $K \geq \operatorname{grp}\left\{H, K^{\prime}\right\}=\Gamma$, a contradiction.

We now construct such an $H$. Fix some open subset $U$, with closure $(U)$ a proper subset of $S^{2}$. Let $m_{1}, m_{2}, \ldots, m_{t}$ be a set of coset representatives for $\Gamma / K_{1}$. Choose some $\varphi_{1}$ with $\partial \varphi_{1} \subset U$. This is done using the same conjugation argument as was employed in 2.6 .

We can suppose that $\partial \varphi_{1}$ is not $\partial m_{1}$ so that the map $m_{1}$ moves $\partial \varphi_{1}$ off itself. Fix a pair of disjoint neighbourhoods of $\partial \varphi_{1}, A$ and $B$, with (a) $A \cup B \subset U$, and (b) $m_{1}(A \cup B)$ disjoint from $A \cup B$. Iterating $\varphi_{1}$ if necessary (and renaming) we can arrange that $A$ and $B$ are absorbing and that $\varphi_{1} \in K_{1}$. Then as in the proof of 2.4, there is a $T$, so that $(A, B)$ is absorbing for the map $\omega_{1}=\varphi_{1}^{T} m_{1} \varphi_{1}^{T}$. Repeating this procedure for each of the $m_{i}$ in turn, with careful choices of the absorbing regions, we arrange a subgroup $\operatorname{grp}\left\{\omega_{1}, \ldots, \omega_{t}\right\}$ with small limit set, and for which $\operatorname{grp}\left\{\omega_{1}, \ldots, \omega_{t}, K_{1}\right\}=\Gamma$.

We can do this for each of the $K_{i}$ in turn, being sure at each stage that the limit set of the $\omega$-subgroup lies inside $U$. The group $H$ is the resulting infinitely generated subgroup.

REMARKS. (a) The question which is raised by the above results is to what extent hyperbolic manifolds have the engulfing property. There are hyperbolic manifolds which have LERF fundamental groups, so examples do exist. Indeed the following conjecture seems possible in the light of the fact that the fundamental group of a finite volume 3-manifold has an especially rich supply of subgroups of finite index due to the failure of the congruence subgroup property [11]:

CONJECTURE. If $\Gamma$ is the fundamental group of a closed, hyperbolic 3-manifold, then it has the engulfing property for subgroups $H$ with $\Lambda H<S^{2}$. 
(b) In the context of free groups a property somewhat stronger than engulfing was introduced in [3]. If $F$ is a finitely generated free group, and $H$ is a subgroup of $F$, then $H$ has a complement in $F$ if there is a subgroup of finite index $K \leq F$ in which $H$ is a free factor. Then one of the results of [3] and [6] is that a finitely generated subgroup of a free group always has a complement. Further, there are several arguments in [4] which show that the existence of a complement implies residual finiteness.

(c) These results have been applied in the case of a totally geodesic surface to deduce that any closed, hyperbolic 3-manifold containing an immersion of a totally geodesic surface is finitely covered by a manifold containing an embedding of a totally geodesic surface $[\mathbf{1 0}]$.

\section{REFERENCES}

1. R. J. B. T. Allenby et al., Frattini subgroups of 3-manifold groups, Trans. Amer. Math. Soc. 247 (1979), 275-299.

2. F. Bonahon, Bouts des variétés hyperboliques de dimension 3, Ann. of Math. 124 (1986), 71158.

3. M. Hall, Coset representations in free groups, Trans. Amer. Math. Soc. 67 (1949), 421-432.

4. J. Hempel, 3-manifolds, Ann. of Math. Studies, no. 86, Princeton Univ. Press, Princeton, N. J.,

5. G. A. Margulis and G. A. Soifer, Maximal subgroups of infinite index in finitely generated linear groups, J. Algebra 69 (1981), 1-23.

6. G. P. Scott, Subgroups of surface groups are almost geometric, J. London Math. Soc. 17 (1978), 555-565; Correction, J. London Math. Soc. 32 (1985), 217-220.

7. H. Bass and J. Morgan, The Smith conjecture, Proc. 1979 Conf., Academic Press, New York,

8. W. P. Thurston, Three dimensional manifolds, Kleinian groups and hyperbolic geometry, Bull. Amer. Math. Soc. 6 (1982), 357-381.

9. __ The geometry and topology of 3-manifolds, Lecture Notes, Princeton, N. J.

10. D. D. Long, Immersions and embeddings of totally geodesic surfaces, Bull. London Math. Soc. 19 (1987), 481-484.

11. A. Lubotzky, Group presentation, p-adic analytic groups and lattices in $S L_{2}(\mathbf{C})$, Ann. of Math. 118 (1983), 115-130.

Department of Mathematics, University of California, Santa Barbara, CALIFORNIA 93106 